\title{
Homotheties of Cylindrically Symmetric Static Manifolds and their Global Extension
}

\author{
Asghar Qadir ${ }^{\dagger *}$, M. Sharif ${ }^{\ddagger}$ and M. Ziad ${ }^{\dagger}$ \\ $\dagger$ Department of Mathematics, \\ Quaid-i-Azam University, Islamabad, Pakistan \\ $\ddagger$ Department of Mathematics, Punjab University \\ Lahore, Pakistan.
}

\begin{abstract}
Cylindrically symmetric static manifolds are classified according to their homotheties and metrics. In each case the homothety vector fields and the corresponding metrics are obtained explicitly by solving the homothety equations. It turns out that these metrics admit homothety groups $H_{m}$, where $m=4,5,7,11$. This classification is then used to identify the cylindrically symmetric static spaces admitting the local homotheties, which are globally prohibited due to their topological construction. Einstein's field equations are then used to identify the physical nature of the spaces thus obtained.
\end{abstract}

PACS numbers: 0240, 0490

Homotheties [1] of metrics are defined to be the vector fields $\mathbf{H}$, along which the metric tensor $(g)$ remains invariant upto constant scale factor $(\phi)$. This amounts to saying that

$$
L_{H} g=2 \phi g
$$

*Senior Associate, Abdus Salam International Centre for Theoretical Physics, Trieste, Italy. 
where $\mathbf{H}$ reduces to an isometry, or a Killing vector field $(\mathrm{KV})$ if $\phi=0$. For $\mathbf{H}$ to be a proper homothety vector field, $\phi \neq 0$. In this paper proper homotheties are used to distinguish the static cylindrically symmetric manifolds which admit $S O(2)$ locally rather than globally and identify the spaces which have topological defects.

In a local coordinate patch, equation (1) is a system of ten partial differential equations (PDEs) involving product terms of first-order partial derivatives of the four components $H^{a}\left(x^{b}\right),(a, b, \ldots=0,1,2,3)$ of the vector field $\mathbf{H}=H^{a} \partial / \partial x^{a}$ and of the ten metric coefficients, all depending in general upon four variables. Due to their physical significance [2], various authors have found self-similar solutions of Einstein's equations by imposing some constraints on the geometry or on the matter content of the spacetimes [3]. Hall and Steele [4] investigated the Segré and Petrov types of spaces that admit proper homothety groups. They classified all such gravitational fields for homothety groups, $H_{m}$, with $m \geq 6$, whereas for $m \leq 5$, some general remarks were given. However, they did not provide the metrics along with the generators of $H_{m}$ explicitly.

An alternative approach [5], which was adopted earlier to obtain complete classifications of manifolds admitting some minimal isometry groups, was further enhanced to obtain the homotheties of spherically symmetric and plane-symmetric static space [6]. Einstein's field equations were then used as the defining equations for the stress-energy tensor. Here the same method is used to obtain the homotheties and the corresponding metrics for cylindrically symmetric static spacetimes (CSSS), written in generalized cylindrical coordinates $\left(x^{0}=c t, x^{1}=\rho, x^{2}=\theta, x^{3}=z\right)$ as [7]

$$
d s^{2}=\exp [\nu(\rho)] d t^{2}-d \rho^{2}-\exp [\lambda(\rho)] a^{2} d \theta^{2}-\exp [\mu(\rho)] d z^{2},
$$

where ' $a$ ' has units of length. Thus the metric admits the group of motions $S O(2) \otimes R^{2}$, where $S O(2)=(\partial / \partial \theta)$, one $R=(\partial / \partial z)$ and the other $R=$ $\left(\partial / \partial_{t}\right)$, as the minimal isometry group. The usual cylindrically symmetric spaces have an axis definable by the limit $\rho \rightarrow 0$. This is analogous to the usual spherically symmetric spaces having an origin as $r \rightarrow 0$. However, there are additional spaces which are classified as spherically symmetric, in that the manifolds have spacelike 2-sections of constant curvature. These are the Bertotti-Robinson and similar metrics. They do not have any origin in the above sense. Correspondingly, for the cylindrically symmetric spaces, there are those which admit the required group $S O(2) \otimes R^{2}$ but do not have 
an axis. We will call these 'cylindrical Bertotti-Robinson metrics'. For the usual cylindrically symmetric manifolds, the difference between an $S O(2)$ and an $R$ is easily seen. For the cylindrical Bertotti-Robinson metrics, it is not a priori clear how to distinguish between them. Using homotheties we are able to distinguish them.

The metric (2) with $\nu=\lambda=\mu=0, a \theta$ replaced by $y$ and $\rho$ by $x$ can easily be recognized as the Minkowski space which was called the 'wrapped Minkowski space' in the classification of CSSS according to their isometries [8]. In fact, the Minkowski space written in cylindrical coordinates acquired special interest with its application 11 to 'topological field theories'. This metric could alternatively be identified as a CSSS, having a line singularity at $\theta=0,2 \pi$, with a topological defect.

Equations (1) written in local coordinates,

$$
\left(H_{a b}\right): g_{a b, c} H^{c}+g_{a c} H_{, b}^{c}+g_{b c} H_{, a}^{c}=2 \phi g_{a b},
$$

are solved for the metric (2) to obtain the required classification. Comparing $\left(H_{i 2}\right)_{, 3}$ and $\left(H_{i 3}\right)_{, 2}(i=0,1)$, using $\left(H_{23}\right)$ and $\left(H_{13}\right)$, one obtains

$$
\begin{gathered}
H_{, 23}^{0}=H_{, 03}^{2}=H_{, 02}^{3}=0, \\
H_{, 23}^{1}=e^{\mu}\left[\left(\mu^{\prime}-\lambda^{\prime}\right) H_{, 2}^{3}+H_{, 21}^{3}\right]
\end{gathered}
$$

and

$$
\left(e^{(\mu-\lambda) / 2} H_{, 2}^{3}\right)_{, 1}=0,
$$

where ',' denotes differentiation relative to $\rho$. Combining equations (4) and (6), one obtains

$$
\left(e^{(\mu-\lambda) / 2} H_{, 2}^{3}\right)_{, i}=0
$$

which gives

$$
H_{, 2}^{3}=e^{(\mu-\lambda) / 2} f(\theta, z) .
$$

Thus from $\left(H_{23}\right)$

$$
H_{, 3}^{2}=-e^{(\mu-\lambda) / 2} f(\theta, z),
$$

\footnotetext{
${ }^{1}$ The simplest example of a topological field theory constructed on a 'wrapped up' Minkowski space was presented by R Rajaraman at the 2nd BCSPIN Summer School in Physics held at Kathmandu, Nepal in 1991. However, it does not appear either in his write-up of the lectures in [9], or in the standard reference.
} 
where $f(\theta, z)$ is a function of integration. Now, using $\left(H_{13}\right)_{, 2},\left(H_{22}\right)_{, 32},\left(H_{33}\right)_{, 32}$ and equations (8) and (9), yields

$$
f(\theta, z)_{, 22}+\alpha f(\theta, z)=0
$$

and

$$
f(\theta, z)_{, 33}-\beta f(\theta, z)=0,
$$

where $\alpha$ and $\beta$ are separation constants given by

$$
\lambda^{\prime}\left(\lambda^{\prime}-\mu^{\prime}\right) e^{\lambda}=4 \alpha
$$

and

$$
\mu^{\prime}\left(\lambda^{\prime}-\mu^{\prime}\right) e^{\mu}=4 \beta
$$

Considering all possible combinations of $\alpha$ and $\beta$ and using the rest of equation $\left(H_{a b}\right)$, one obtains a complete solution of equation (3), giving $H^{a}$ and the corresponding metric components. These results are listed according to their homothety groups, $H_{m}$, as follows.

There are three metrics admitting four homotheties:

(a)

$$
\begin{aligned}
\nu & =2 \alpha \ln \left(\rho / \rho_{0}\right), \quad \lambda=2 \ln (\rho / a), \quad \mu=0, \quad(\alpha \neq 0,1) \\
H^{0} & =(1-\alpha) \phi t+C_{0}, \quad H_{1}=\rho \phi, \quad H^{2}=C_{2}, \quad H^{3}=\phi z+C_{3}
\end{aligned}
$$

of Petrov type I [8] and Segré type [1,111] [8];

(b)

$$
\begin{aligned}
\nu & =2 \alpha \ln \left(\rho / \rho_{0}\right), \quad \lambda=\mu=2 \beta \ln \left(\rho / \rho_{0}\right), \quad(\alpha \neq \beta \text { and } \alpha, \beta \neq 0,1) \\
H^{0} & =(1-\alpha) \phi t+C_{0}, \quad H_{1}=\rho \phi \\
H^{2} & =(1-\beta) \phi \theta+C_{2}, \quad H^{3}=(1-\beta) \phi z+C_{3}
\end{aligned}
$$

of Petrov type D and Segré type[1,(11)1];

(c)

$$
\begin{aligned}
\nu & =2 \alpha \ln \left(\rho / \rho_{0}\right), \quad \lambda=0, \quad \mu=2 \ln \left(\rho / \rho_{0}\right), \\
H^{0} & =(1-\alpha) \phi, \quad H^{1}=\rho \phi, \quad H^{2}=\phi \theta+C_{2}, \quad H^{3}=C_{3},
\end{aligned}
$$

of Petrov type I and Segré type $[1,111]$.

There are three metrics admitting five homotheties, which are: 
(a)

$$
\begin{aligned}
\nu & =2 \ln \left(\rho / \rho_{0}\right), \quad \lambda=2 \ln (\rho / a), \quad \mu=0, \\
H^{0} & =C_{1} \theta+C_{0}, \quad H^{1}=\rho \phi, \\
H^{2} & =\left(C_{1} / \rho_{0}^{2}\right) t+C_{3}, \quad H^{3}=\phi z+C_{3},
\end{aligned}
$$

of Petrov type D, Segré type $[1,111]$;

(b)

$$
\begin{aligned}
\nu & =\mu=2 \ln \left(\rho / \rho_{0}\right), \quad \lambda=0, \\
H^{0} & =z C_{1}+C_{0}, \quad H^{1}=\rho \phi, \\
H^{2} & =\phi \theta+C_{3}, \quad H^{3}=C_{1} t+C_{3},
\end{aligned}
$$

of Petrov type D, Segré type [1,111],

(c)

$$
\begin{aligned}
\nu & =0, \quad \lambda=2 \ln (\rho / a)=\mu, \\
H^{0} & =\phi t+C_{0}, \quad H^{1}=\rho \phi, \\
H^{2} & =(-z / a) C_{4}+C_{2}, \quad H^{3}=a \theta C_{4}+C_{3},
\end{aligned}
$$

of Petrov type D, Segré type [(1,1)(11)].

There is only one metric admitting seven homotheties:

$$
\begin{aligned}
\nu & =\lambda=\mu=2 \alpha \ln \left(\rho / \rho_{0}\right), \quad(\alpha \neq 0,1) \\
H^{0} & =C_{1} z+\alpha \theta C_{3}+(1-\alpha) \phi t+C_{0}, \\
H^{1} & =\rho \phi, \\
H^{2} & =(-z / a) C_{4}+(1-\alpha) \phi \theta+(t / a) C_{3}+C_{6}, \\
H^{3} & =a \theta C_{4}+(1-\alpha) \phi z+C_{1} t+C_{2},
\end{aligned}
$$

of Petrov type D, Segré type [1,(11)1].

There are three metrics admitting 11 homotheties, other than proper Minkowski space which admits $\mathbf{H}=\phi(t \partial / \partial t+\rho \partial / \partial \rho+z \partial / \partial z)$ as a proper homothety vector. They are:

(a) $\quad \nu=\lambda=0, \quad \mu=2 \ln \left(\rho / \rho_{0}\right) \quad$ with

$$
\mathbf{H}=\phi(t \partial / \partial t+\rho \partial / \partial \rho+\theta \partial / \partial \theta)+z \partial / \partial z),
$$

(b) $\nu=\lambda=\mu=0, \quad$ with $\quad \mathbf{H}=\phi(\mathrm{t} \partial / \partial \mathrm{t}+\rho \partial / \partial \rho+\theta \partial / \partial \theta+\mathrm{z} \partial / \partial$ 女 $\& 2)$

(c) $\nu=2 \ln \left(\rho / \rho_{0}\right), \quad \lambda=\mu=0 \quad$ with

$$
\mathbf{H}=\phi(\rho \partial / \partial \rho+\theta \partial / \partial \theta+z \partial / \partial z) \text {. }
$$


In equations (14)-(23), there are two types of solutions: one in which the $H^{2}$ component of the proper homothety vector field is zero; and the other in which it is non-zero and involves $\theta$. This shows that in the first type of solution, the homotheties can be extended globally, whereas in the latter this is not possible due to a line singularity at $\theta=0,2 \pi$. This is how one can identify the topologically constructed spaces, even if they are non-flat.

As a result of this classification, we see that there exist cases of four, five, seven and 11 homotheties. The metrics given by equations (21)-(23); equation (20); equation (18) and equations (15) and (16) admitting 11, seven, five and four homotheties, respectively, do not appear in the classification of Hall and Steele [4] due to their topological construction, as these admit homotheties which cannot be extended globally. However, the spaces other than the proper Minkowski space given by equations (14) and (17), where the homothety vector fields could be globally extended, match their solution. The metric given by equation (19) can be considered to be that of equation (17) written in different coordinates.

The metric given by equation (15) represents a perfect fluid for $\beta=$ $\alpha(\alpha-1) /(\alpha+1)$, whereas it represents a non-null electromagnetic field for

$\beta=\alpha+1$. Both conditions are satisfied when $\alpha=-\frac{1}{3}$ and $\beta=\frac{2}{3}$. The metric given by equation (19) represents a non-null electromagnetic field. The metric given by equation (20) represents a tachyonic fluid and could be reinterpreted as an anisotropic fluid with an appropriately chosen cosmological constant. All other metrics are non-physical. Of course, any non-physical metric could be re-interpreted as a physical metric with an appropriately chosen cosmological constant. However, these metrics are not of particular interest.

\section{Acknowledgements}

We are extremely grateful to the referees for their highly useful comments. Two of the authors (AQ and MZ) would like to thank Professor M. Virasoro and the Abdus Salam ICTP for hospitality while this work was being done. One of the authors (MS) is grateful to the Third World Academy of Sciences Associateship Scheme for support to travel to Brazil and to the State University of Campinas for local hospitality, while the work was being done. This work was partially supported under Pakistan Science Foundation Project PSF/RES-C-QU/MATHS(21). 


\section{References}

[1] Katzin G.H., Levine J. and Davis W.R. J. Math. Phys. 10(1969)617;

Katzin G.H., Levine J. and Davis W.R. J. Math. Phys. 11(1970)1518.

[2] Cahill M.E. and Taub A.H. Commun. Math. Phys. 21(1971)1;

Taub A.H. General Relativity ed L O' Raifertaigh (Oxford: Oxford University Press, 1972)p 135-50;

Winicour J. Lecture Notes in Physics vol 14 (Berlin: Springer, 1972)p 145;

Eardley D.M. Commun, Math, Phys. 37(1974)287;

Mclntosh C.B.G. Phys. Lett. A50(1975)429;

Mclntosh C.B.G. Gen. Rel. Grav. 7(1976)199;

Mclntosh C.B.G. Gen. Rel. Grav. 7(1976)215.

[3] Yano K. J. Indian Math. Soc. (NS) 15(1951)105;

Ehlers J. and Kundt W. Gravitation: An Introduction to Current Research ed L. Witten (New York: Wiley 1962)p 80;

Collinson C.D. and French D.C. J. Math. Phys. 8(1967)701;

Godfrey B.B. Gen. Rel. Grav. 3(1972)3;

Thompson A. Phys. Rev. Lett. 349(1975)507;

Halford W.D. and Kerr R.P. J. Math. Phys. 21(1980)120;

Halford W.D. J. Math. Phys. 21(1980)129;

Hall G.S. Gen. Rel. Grav. 20(1988)671.

[4] Hall G.S. and Steele J.D. Gen. Rel. Grav. 22(1990)457.

[5] Bokhari A.H. and Qadir A. J. Math. Phys. 28(1987)1019;

Bokhari A.H. and Qadir A. J. Math. Phys. 31(1990)1463;

Qadir A. and Ziad M. J. Math. Phys. 29(1988)2473;

Qadir A. and Ziad M. J. Math. Phys. 31(1989)254;

Qadir A. and Ziad M. Nuovo Cimento B110(1995)317;

Qadir A. and Ziad M. Proc. 6th Marcel Grossmann Meeting ed T. Nakamura and H. Sato (Singapore: World Scientific, 1993)p 1115-8.

[6] Ahmad D. and Ziad M. J. Math. Phys. 38(1997)2547;

Ziad M. The classification of static plane symmetric spacetimes Nuovo Cimento B114 (to appear). 
[7] Kramers D., Stephani H., MacCallum M.A.H. and Herlt E. Exact Solutions of Einsterin's Field Equations (Berlin: Deutscher, 1980).

[8] Qadir A. and Ziad M. Nuovo Cimento B110(1995)277.

[9] Rajaraman R. Current Topics in Condensed matter and Particle Physics ed J.C. Pati, Q. Shafi and Y. Lu (Singapore: World Scientific, 1993). 